A note on two-dimensional systems of linear differential inequalities with argument deviations

Jiřı Šremr 


\title{
A NOTE ON TWO-DIMENSIONAL SYSTEMS OF LINEAR DIFFERENTIAL INEQUALITIES WITH ARGUMENT DEVIATIONS
}

\author{
JIŘÍ ŠREMR
}

[Received: 8 March, 2006]

\begin{abstract}
In this paper, the question on the positivity of the Cauchy operator of two-dimensional systems of differential equations with argument deviations is studied. Some results of [11] are refined for two-dimensional systems of differential inequalities.
\end{abstract}

Mathematics Subject Classification: 34K06, 34K10

Keywords: System of diferential equations with argument deviations, initial value problem, sign-constant solutions, theorem on differential inequalities

\section{NOTATION AND INTRODUCTION}

On the interval $[a, b]$, we consider two-dimensional differential system

$$
u_{i}^{\prime}(t)=p_{i 1}(t) u_{1}\left(\tau_{i 1}(t)\right)+p_{i 2}(t) u_{2}\left(\tau_{i 2}(t)\right)+q_{i}(t) \quad(i=1,2)
$$

with the initial conditions

$$
u_{1}(a)=c_{1}, \quad u_{2}(a)=c_{2},
$$

where $p_{i k}, q_{i}:[a, b] \rightarrow \mathbb{R}(i, k=1,2)$ are Lebesgue integrable functions, $\tau_{i k}:$ $[a, b] \rightarrow[a, b](i, k=1,2)$ are measurable functions, and $c_{1}, c_{2} \in \mathbb{R}$. By a solution of the problem (1.1), (1.2), we understand an absolutely continuous vector function $u=\left(u_{1}, u_{2}\right)^{T}:[a, b] \rightarrow \mathbb{R}^{2}$ satisfying the equation (1.1) almost everywhere on $[a, b]$ and verifying the initial conditions (1.2). The following notation is used throughout the paper:

$\mathbb{R}$ is the set of all real numbers, $\mathbb{R}_{+}=[0,+\infty[$;

$\mathbb{R}^{2}$ is the space two-dimensional column vectors $x=\left(x_{i}\right)_{i=1}^{2}$ with the elements $x_{1}, x_{2} \in \mathbb{R}$ and the norm

$$
\|x\|=\left|x_{1}\right|+\left|x_{2}\right|
$$

The research was supported by the Grant Agency of the Czech Republic, Grant No. 201/04/P183, and by the Academy of Sciences of the Czech Republic, Institutional Research Plan No. AV0Z10190503. 
If $x, y \in \mathbb{R}^{2}$ then

$x \leq y \quad$ if and only if $\quad x_{1} \leq y_{1}, x_{2} \leq y_{2} ;$

$\mathbb{R}^{2 \times 2}$ is the space of the $2 \times 2$-matrices $X=\left(x_{i k}\right)_{i, k=1}^{2}$ with the elements $x_{i k} \in \mathbb{R}$ $(i, k=1,2)$;

$r(X)$ is the spectral radius of the matrix $X \in \mathbb{R}^{2 \times 2}$;

$X^{T}$ is the transposed matrix to $n \times m$-matrix $X$;

If $x_{1}, x_{2} \in \mathbb{R}$ then

$$
\operatorname{diag}\left(x_{1}, x_{2}\right)=\left(\begin{array}{cc}
x_{1} & 0 \\
0 & x_{2}
\end{array}\right)
$$

$C\left([a, b] ; \mathbb{R}^{2}\right)$ is the Banach space of continuous vector functions $u:[a, b] \rightarrow \mathbb{R}^{2}$ equipped with the norm

$$
\|u\|_{C}=\max \{\|u(t)\|: t \in[a, b]\} ;
$$

$\widetilde{C}\left([a, b] ; \mathbb{R}^{2}\right)$ is the set of absolutely continuous vector functions $u:[a, b] \rightarrow \mathbb{R}^{2}$; $L\left([a, b] ; \mathbb{R}^{2}\right)$ is the Banach space of Lebesgue integrable vector functions $h$ : $[a, b] \rightarrow \mathbb{R}^{2}$ equipped with the norm

$$
\|h\|_{L}=\int_{a}^{b}\|h(s)\| d s
$$

The equalities and inequalities with integrable functions are understood to hold almost everywhere.

The following proposition is well-known.

Proposition 1.1. Let

$$
p_{i 3-i}(t) \geq 0 \quad \text { for } t \in[a, b], i=1,2 .
$$

Then, for every vector function $\left(\gamma_{1}, \gamma_{2}\right)^{T} \in \widetilde{C}\left([a, b] ; \mathbb{R}^{2}\right)$ satisfying

$$
\begin{gathered}
\gamma_{i}^{\prime}(t) \leq \sum_{k=1}^{2} p_{i k}(t) \gamma_{k}(t)+q_{i}(t) \quad \text { for } t \in[a, b], i=1,2, \\
\gamma_{1}(a) \leq c_{1}, \quad \gamma_{2}(a) \leq c_{2},
\end{gathered}
$$

the relation

$$
\gamma_{i}(t) \leq u_{i}(t) \quad \text { for } t \in[a, b], i=1,2
$$

holds, where $\left(u_{1}, u_{2}\right)^{T}$ is a solution of the system

$$
u_{i}^{\prime}=\sum_{k=1}^{2} p_{i k}(t) u_{k}+q_{i}(t) \quad(i=1,2)
$$

satisfying the initial conditions (1.2). 
In the other words, if the condition (1.3) is satisfied then so-called theorem on differential inequalities holds for the system (1.4) or, equivalently, the Cauchy matrix function of the homogeneous system corresponding to (1.4) is non-negative. It is very easy to show that, under the assumption (1.3), an analogous assertion for the system (1.1) does not hold in general. Consequently, some stronger assumptions have to be required for differential systems with argument deviations. There are a lot of results concerning various types of theorems on functional differential inequalities. Let us mention, among other, papers [1-4, 6, 8, 9]. We have studied this question in [11].

Following [11], we introduce a definition.

Definition 1.1. Let $\sigma_{1}, \sigma_{2} \in\{-1,1\}$. We say that a linear bounded operator $\ell$ : $C\left([a, b] ; \mathbb{R}^{2}\right) \rightarrow L\left([a, b] ; \mathbb{R}^{2}\right)$ belongs to the set $\delta_{a b}^{2,\left(\sigma_{1}, \sigma_{2}\right)}(a)$ if every vector function $u \in \widetilde{C}\left([a, b] ; \mathbb{R}^{2}\right)$ such that

$$
\begin{gathered}
\operatorname{diag}\left(\sigma_{1}, \sigma_{2}\right)\left[u^{\prime}(t)-\ell(u)(t)\right] \geq 0 \quad \text { for } t \in[a, b], \\
\operatorname{diag}\left(\sigma_{1}, \sigma_{2}\right) u(a) \geq 0
\end{gathered}
$$

satisfies the conditions

$$
\sigma_{i} u_{i}(t) \geq 0 \quad \text { for } t \in[a, b], i=1,2 .
$$

Remark 1.1. Let the operator $\ell: C\left([a, b] ; \mathbb{R}^{2}\right) \rightarrow L\left([a, b] ; \mathbb{R}^{2}\right)$ be defined by the relation

$$
\begin{aligned}
\ell(v)(t) \stackrel{\text { def }}{=}\left(\begin{array}{l}
p_{11}(t) v_{1}\left(\tau_{11}(t)\right)+p_{12}(t) v_{2}\left(\tau_{12}(t)\right) \\
p_{21}(t) v_{1}\left(\tau_{21}(t)\right)+p_{22}(t) v_{2}\left(\tau_{22}(t)\right)
\end{array}\right) \\
\text { for } t \in[a, b], v=\left(v_{1}, v_{2}\right)^{T} \in C\left([a, b] ; \mathbb{R}^{2}\right)
\end{aligned}
$$

and let $\ell \in 8_{a b}^{2,\left(\sigma_{1}, \sigma_{2}\right)}(a)$. Then it is easy to verify that the homogeneous problem corresponding to (1.1), (1.2) has only the trivial solution. Therefore, according to the Fredholm property of the linear boundary value problems for functional differential systems (see, e. g., $[5-7,10])$, the problem $(1.1),(1.2)$ has a unique solution for any $\left(q_{1}, q_{2}\right)^{T} \in L\left([a, b] ; \mathbb{R}^{2}\right)$ and $c_{1}, c_{2} \in \mathbb{R}$. However, the inclusion $\ell \in \delta_{a b}^{2,\left(\sigma_{1}, \sigma_{2}\right)}(a)$ guarantees, in addition, that the solution $\left(u_{1}, u_{2}\right)^{T}$ of the problem indicated satisfies

$$
\sigma_{i} u_{i}(t) \geq 0 \text { for } t \in[a, b], i=1,2
$$

if the relations

$$
\sigma_{i} q_{i}(t) \geq 0 \quad \text { for } t \in[a, b], \quad \sigma_{i} c_{i} \geq 0 \quad(i=1,2)
$$

are true.

In the sequel, we set

$$
\tau^{*}=\max \left\{\operatorname{ess} \sup \left\{\tau_{i k}(t): t \in[a, b]\right\}: i, k=1,2\right\} .
$$

The following proposition is given in [11]. 
Proposition 1.2 ([11, Theorem 4.1]). Let $\sigma_{1}, \sigma_{2} \in\{-1,1\}$ and let the functions $p_{j k}(j, k=1,2)$ satisfy the conditions

$$
\sigma_{j} \sigma_{k} p_{j k}(t) \geq 0 \quad \text { for } t \in[a, b], j, k=1,2 .
$$

Let, moreover, there exist numbers $\delta_{i}>0(i=1,2)$ such that

$$
\max \left\{\frac{1}{\delta_{i}} \sum_{k=1}^{2} \delta_{k} \int_{a}^{\tau^{*}}\left|p_{i k}(s)\right| d s: i=1,2\right\}=1
$$

and $r(A)<1$, where the matrix $A=\left(a_{i k}\right)_{i, k=1}^{2}$ is given by the equality

$$
a_{i k}=\frac{\delta_{k}}{\delta_{i}} \sum_{j=1}^{2} \int_{a}^{\tau^{*}}\left|p_{i j}(s)\right|\left(\int_{a}^{\tau_{i j}(s)}\left|p_{j k}(\xi)\right| d \xi\right) d s \quad \text { for } i, k=1,2 .
$$

Then the operator $\ell$ defined by (1.8) belongs to the set $\delta_{a b}^{2,\left(\sigma_{1}, \sigma_{2}\right)}(a)$.

We have also shown in [11] (see Example 5.3) that the assumption $r(A)<1$ in the last proposition is optimal and cannot be weakened. On the other hand, the following example shows that the assumption indicated is not necessary for the assertion of Proposition 1.2.

Example 1.1. Let $p_{i i} \equiv 0(i=1,2)$ and let $p_{12}, p_{21}:[a, b] \rightarrow \mathbb{R}_{+}$be integrable functions such that

$$
\int_{a}^{b} p_{12}(s) d s=1, \quad \int_{a}^{b} p_{21}(s) d s=1 .
$$

It is clear that there exists $t_{0} \in[a, b]$ satisfying

$$
\int_{a}^{t_{0}} p_{12}(s) d s<1
$$

Put $\delta_{1}=1, \delta_{2}=1, \tau_{i i} \equiv a(i=1,2), \tau_{12} \equiv b$, and $\tau_{21} \equiv t_{0}$. Then, the matrix $A=\left(a_{i k}\right)_{i, k=1}^{2}$ given by (1.12) has the form

$$
A=\left(\begin{array}{cc}
\int_{a}^{b} p_{12}(s) d s \int_{a}^{b} p_{21}(s) d s & 0 \\
0 & \int_{a}^{b} p_{21}(s) d s \int_{a}^{t_{0}} p_{12}(s) d s
\end{array}\right)
$$

It is clear that, by virtue of (1.13) and (1.14), we have $r(A)=1$.

According to (1.13), the condition (1.11) is satisfied. Therefore, it follows from Proposition 3.3 of [11] that the operator $\ell$ given by (1.8) belongs to the set $\delta_{a b}^{2,(1,1)}(a)$ if and only if the homogeneous problem corresponding to (1.1), (1.2) has only the trivial solution. Let $u=\left(u_{1}, u_{2}\right)^{T}$ be a solution of the problem indicated. Then

$$
u_{1}^{\prime}(t)=p_{12}(t) u_{2}(b), \quad u_{2}^{\prime}(t)=p_{21}(t) u_{1}\left(t_{0}\right) \quad \text { for } t \in[a, b],
$$




$$
u_{1}(a)=0, \quad u_{2}(a)=0 .
$$

The integration of the second equality in (1.15) from $a$ to $b$, in view of (1.13) and (1.16), results in

$$
u_{2}(b)=u_{1}\left(t_{0}\right) \int_{a}^{b} p_{21}(s) d s=u_{1}\left(t_{0}\right) .
$$

On the other hand, the integration of the first equality in (1.15) from $a$ to $t_{0}$, on account of (1.16), implies

$$
u_{1}\left(t_{0}\right)=u_{2}(b) \int_{a}^{t_{0}} p_{12}(s) d s=u_{1}\left(t_{0}\right) \int_{a}^{t_{0}} p_{12}(s) d s .
$$

Hence, using (1.14) in the last relations, we get $u_{1}\left(t_{0}\right)=0$ and thus $u_{2}(b)=0$, as well. Consequently, (1.15) and (1.16) yield $u_{1} \equiv 0$ and $u_{2} \equiv 0$.

Therefore, the operator $\ell$ defined by (1.8) belongs to the set $\delta_{a b}^{2,(1,1)}(a)$ even if $r(A)=1$.

In Section 3, we shall give efficient conditions which are not only sufficient but also necessary for the inclusion $\ell \in \delta_{a b}^{2,\left(\sigma_{1}, \sigma_{2}\right)}(a)$ with $\ell$ given by (1.8) provided that there exist numbers $\delta_{i}>0(i=1,2)$ such that (1.10) and (1.11) are satisfied.

\section{AUXiliary STATEMENTS}

In addition to (1.9), we put

$$
\tau_{i i}^{*}=\operatorname{ess} \sup \left\{\tau_{i i}(t): t \in[a, b]\right\} \quad \text { for } i=1,2 .
$$

To prove the main results (see Section 3 ) we need some auxiliary statements. Following [4], we introduce a definition.

Definition 2.1. Let $C([a, b] ; \mathbb{R})$ and $L([a, b] ; \mathbb{R})$ denote the Banach spaces of continuous and Lebesgue integrable functions $z:[a, b] \rightarrow \mathbb{R}$, respectively, equipped with the standard norms. We say that a linear bounded operator $\varphi: C([a, b] ; \mathbb{R}) \rightarrow$ $L([a, b] ; \mathbb{R})$ belongs to the set $\delta_{a b}(a)$ if every absolutely continuous function $z$ : $[a, b] \rightarrow \mathbb{R}$ such that

$$
z^{\prime}(t) \geq \varphi(z)(t) \quad \text { for } t \in[a, b], \quad z(a) \geq 0
$$

is non-negative on $[a, b]$.

For $i=1,2$, we put

$$
\ell_{i i}(z)(t) \stackrel{\text { def }}{=} p_{i i}(t)\left(\tau_{i i}(t)\right) \quad \text { for } t \in[a, b], z \in C([a, b] ; \mathbb{R}) .
$$

Lemma 2.1 ([11, Proposition 3.1]). Let $\sigma_{1}, \sigma_{2} \in\{-1,1\}$, the condition (1.10) be satisfied, and let

$$
\int_{a}^{\tau^{*}} p_{12}(s) d s \int_{a}^{\tau^{*}} p_{21}(s) d s=0 .
$$


Then the operator $\ell$ given by (1.8) belongs to the set $8_{a b}^{2,\left(\sigma_{1}, \sigma_{2}\right)}(a)$ if and only if both operators $\ell_{11}$ and $\ell_{22}$ defined by (2.2) belong to the set $\delta_{a b}(a)$.

Lemma 2.2. Let $i \in\{1,2\}$ and

$$
p_{i i}(t) \geq 0 \quad \text { for } t \in[a, b] .
$$

Then the following assertions are true:

(a) If

$$
\int_{a}^{\tau_{i i}^{*}} p_{i i}(s) d s<1
$$

then the operator $\ell_{i i}$ defined by (2.2) belongs to the set $\delta_{a b}(a)$.

(b) Let

$$
\int_{a}^{\tau_{i i}^{*}} p_{i i}(s) d s=1
$$

Then the operator $\ell_{i i}$ defined by (2.2) belongs to the set $\S_{a b}(a)$ if and only if

$$
\int_{a}^{\tau_{i i}^{*}} p_{i i}(s)\left(\int_{a}^{\tau_{i i}(s)} p_{i i}(\xi) d \xi\right) d s<1 .
$$

The results of the last lemma are partly contained in [4]. For the sake of completeness, we give the proof here.

Proof of LEMma 2.2. Let $C\left(\left[a, \tau_{i i}^{*}\right] ; \mathbb{R}\right)$ be the Banach space of the continuous functions $z:\left[a, \tau_{i i}^{*}\right] \rightarrow \mathbb{R}$ equipped with the standard norm. Let the operator $\ell_{i i}$ be given by (2.2) and let

$$
\ell_{i i}^{*}(z)(t) \stackrel{\text { def }}{=} p_{i i}(t) z\left(\tau_{i i}(t)\right) \quad \text { for } t \in\left[a, \tau_{i i}^{*}\right], z \in C\left(\left[a, \tau_{i i}^{*}\right] ; \mathbb{R}\right) .
$$

In the other words, $\ell_{i i}^{*}$ is the restriction of $\ell_{i i}$ to the space $C\left(\left[a, \tau_{i i}^{*}\right] ; \mathbb{R}\right)$. Since the condition (2.4) holds and

$$
\tau_{i i}(t) \leq \tau_{i i}^{*} \quad \text { for } t \in[a, b],
$$

it is clear that $\ell_{i i} \in \delta_{a b}(a)$ if and only if $\ell_{i i}^{*} \in \delta_{a \tau_{i i}^{*}}(a)$.

Case (a). Let the condition (2.5) be satisfied. By virtue of Remark 1.1 in [4], we find $\ell_{i i}^{*} \in \oiint_{a \tau_{i i}^{*}}(a)$, and thus $\ell_{i i} \in \oiint_{a b}(a)$.

Case (b). Let the condition (2.6) be fulfilled. According to Remark 1.1 in [4], $\ell_{i i}^{*} \in S_{a \tau_{i i}^{*}}(a)$ if and only if the homogeneous problem

$$
\begin{gathered}
z^{\prime}(t)=p_{i i}(t) z\left(\tau_{i i}(t)\right) \quad\left(t \in\left[a, \tau_{i i}^{*}\right]\right), \\
z(a)=0
\end{gathered}
$$


has only the trivial solution*. Consequently, to prove the lemma it is sufficient to show that the homogeneous problem (2.8), (2.9) has only the trivial solution if and only if the condition (2.7) is satisfied.

Let $z$ be a solution of the problem (2.8), (2.9). Put

$$
M=\max \left\{z(t): t \in\left[a, \tau_{i i}^{*}\right]\right\}, \quad m=\min \left\{z(t): t \in\left[a, \tau_{i i}^{*}\right]\right\}
$$

and choose $t_{M}, t_{m} \in\left[a, \tau_{i i}^{*}\right]$ such that

$$
z\left(t_{M}\right)=M, \quad z\left(t_{m}\right)=m .
$$

Obviously, (2.9) and (2.10) imply

$$
M \geq 0 \text {. }
$$

We can assume without loss of generality that $t_{m} \leq t_{M}$. The integration of (2.8) from $t_{m}$ to $t_{M}$, in view of (2.4), (2.6), and (2.10)-(2.12), yields

$$
M-m=\int_{t_{m}}^{t_{M}} p_{i i}(s) z\left(\tau_{i i}(s)\right) d s \leq M \int_{t_{m}}^{t_{M}} p_{i i}(s) d s \leq M .
$$

Hence we get $m \geq 0$, i. e.,

$$
z(t) \geq 0 \quad \text { for } t \in\left[a, \tau_{i i}^{*}\right]
$$

From (2.4), (2.8), and (2.13) we obtain

$$
z(t) \leq z\left(\tau_{i i}^{*}\right) \quad \text { for } t \in\left[a, \tau_{i i}^{*}\right]
$$

Put

$$
f(t)=\int_{a}^{t} p_{i i}(s) d s \quad \text { for } t \in\left[a, \tau_{i i}^{*}\right] .
$$

The integration of (2.8) from $t$ to $\tau_{i i}^{*}$, on account of (2.4) and (2.14), yields

$$
z\left(\tau_{i i}^{*}\right)-z(t)=\int_{t}^{\tau_{i i}^{*}} p_{i i}(s) z\left(\tau_{i i}(s)\right) d s \leq z\left(\tau_{i i}^{*}\right) \int_{t}^{\tau_{i i}^{*}} p_{i i}(s) d s \quad \text { for } t \in\left[a, \tau_{i i}^{*}\right] .
$$

Using (2.6), (2.15) and the last relations, we get

$$
z\left(\tau_{i i}^{*}\right) f(t)=z\left(\tau_{i i}^{*}\right)\left(1-\int_{t}^{\tau_{i i}^{*}} p_{i i}(s) d s\right) \leq z(t) \quad \text { for } t \in\left[a, \tau_{i i}^{*}\right] .
$$

On the other hand, the integration of (2.8) from $a$ to $t$, on account of (2.4), (2.9), (2.14), and (2.15), results in

$$
z(t)=\int_{a}^{t} p_{i i}(s) z\left(\tau_{i i}(s)\right) d s \leq z\left(\tau_{i i}^{*}\right) \int_{a}^{t} p_{i i}(s) d s=z\left(\tau_{i i}^{*}\right) f(t) \quad \text { for } t \in\left[a, \tau_{i i}^{*}\right] .
$$

Now, from the last relations and (2.16) we obtain

$$
z(t)=z\left(\tau_{i i}^{*}\right) f(t) \quad \text { for } t \in\left[a, \tau_{i i}^{*}\right] .
$$

*By a solution of the problem (2.8), (2.9), we mean an absolutely continuous function $z:\left[a, \tau_{i i}^{*}\right] \rightarrow$ $\mathbb{R}$ satisfying the equation (2.8) almost everywhere on $\left[a, \tau_{i i}^{*}\right]$ and verifying also the initial condition (2.9). 
Finally, the integration of (2.8) from $a$ to $\tau_{i i}^{*}$, with respect to (2.9) and (2.17), implies

$$
z\left(\tau_{i i}^{*}\right)=\int_{a}^{\tau_{i i}^{*}} p_{i i}(s) z\left(\tau_{i i}(s)\right) d s=z\left(\tau_{i i}^{*}\right) \int_{a}^{\tau_{i i}^{*}} p_{i i}(s) f\left(\tau_{i i}(s)\right) d s,
$$

whence we get

$$
z\left(\tau_{i i}^{*}\right)\left[1-\int_{a}^{\tau_{i i}^{*}} p_{i i}(s)\left(\int_{a}^{\tau_{i i}(s)} p_{i i}(\xi) d \xi\right) d s\right]=0 .
$$

We have proved that every solution $z$ of the problem (2.8), (2.9) admits the representation (2.17), where $z\left(\tau_{i i}^{*}\right)$ satisfies (2.18). Consequently, if (2.7) holds then the homogeneous problem $(2.8),(2.9)$ has only the trivial solution.

It remains to show that if (2.7) is not satisfied, i. e.,

$$
\int_{a}^{\tau_{i i}^{*}} p_{i i}(s) f\left(\tau_{i i}(s)\right) d s=1
$$

then the homogeneous problem (2.8), (2.9) has a non-trivial solution. Indeed, in view of (2.4) and (2.6), (2.15) yields

$$
f(t) \leq f\left(\tau_{i i}^{*}\right)=1 \quad \text { for } t \in\left[a, \tau_{i i}^{*}\right]
$$

Therefore, using (2.4) and (2.19), it is easy to verify that

$$
\begin{aligned}
0 \leq \int_{a}^{t} p_{i i}(s)\left[1-f\left(\tau_{i i}(s)\right)\right] d s \leq \int_{a}^{\tau_{i i}^{*}} p_{i i}(s)\left[1-f\left(\tau_{i i}(s)\right)\right] d s= \\
=1-\int_{a}^{\tau_{i i}^{*}} p_{i i}(s) f\left(\tau_{i i}(s)\right) d s=0 \text { for } t \in\left[a, \tau_{i i}^{*}\right],
\end{aligned}
$$

whence we get

$$
f(t)=\int_{a}^{t} p_{i i}(s) f\left(\tau_{i i}(s)\right) d s \quad \text { for } t \in\left[a, \tau_{i i}^{*}\right]
$$

Consequently, $f$ is a non-trivial solution of the problem (2.8), (2.9).

Lemma 2.3. Let $\sigma_{1}, \sigma_{2} \in\{-1,1\}$, the condition (1.10) hold, and let there exist numbers $\delta_{j}>0(j=1,2)$ such that the relation

$$
\delta_{1} \int_{a}^{\tau^{*}}\left|p_{i 1}(s)\right| d s+\delta_{2} \int_{a}^{\tau^{*}}\left|p_{i 2}(s)\right| d s=\delta_{i}
$$

is satisfied for $i=1,2$. Let, moreover, $u=\left(u_{1}, u_{2}\right)^{T}$ be a solution of the homogeneous problem

$$
\begin{gathered}
u_{i}^{\prime}(t)=p_{i 1}(t) u_{1}\left(\tau_{i 1}(t)\right)+p_{i 2}(t) u_{2}\left(\tau_{i 2}(t)\right) \quad\left(t \in\left[a, \tau^{*}\right], i=1,2\right) \\
u_{1}(a)=0, \quad u_{2}(a)=0
\end{gathered}
$$


Then both functions $u_{1}$ and $u_{2}$ do not change their signs on $\left[a, \tau^{*}\right]$. If, in addition,

$$
\int_{a}^{\tau^{*}}\left|p_{12}(s)\right| d s+\int_{a}^{\tau^{*}}\left|p_{21}(s)\right| d s>0
$$

then the relation

$$
\sigma_{1} \sigma_{2} u_{1}(t) u_{2}(t) \geq 0 \quad \text { for } t \in\left[a, \tau^{*}\right]
$$

is satisfied.

Proof. For $i=1,2$, we put

$$
M_{i}=\max \left\{\sigma_{i} u_{i}(t): t \in\left[a, \tau^{*}\right]\right\}, \quad m_{i}=-\min \left\{\sigma_{i} u_{i}(t): t \in\left[a, \tau^{*}\right]\right\} .
$$

Choose $t_{i}, T_{i} \in\left[a, \tau^{*}\right](i=1,2)$ such that

$$
\sigma_{i} u_{i}\left(T_{i}\right)=M_{i}, \quad \sigma_{i} u_{i}\left(t_{i}\right)=-m_{i} \quad \text { for } i=1,2,
$$

and put

$$
\tilde{p}_{i k}=\int_{a}^{\tau^{*}}\left|p_{i k}(s)\right| d s \quad \text { for } i, k=1,2 .
$$

Let us first suppose that both functions $u_{1}$ and $u_{2}$ change their signs on $\left[a, \tau^{*}\right]$. Then we have

$$
M_{i}>0, \quad m_{i}>0
$$

for $i=1,2$. We can assume without loss of generality that $T_{1}<t_{1}$. The integration of (2.21) with $i=1$ from $T_{1}$ to $t_{1}$, in view of (1.10) and (2.25)-(2.27), yields

$$
\begin{array}{r}
M_{1}+m_{1}=-\sigma_{1} \int_{T_{1}}^{t_{1}} p_{11}(s) u_{1}\left(\tau_{11}(s)\right) d s-\sigma_{1} \int_{T_{1}}^{t_{1}} p_{12}(s) u_{2}\left(\tau_{12}(s)\right) d s \leq \\
\leq m_{1} \int_{T_{1}}^{t_{1}}\left|p_{11}(s)\right| d s+m_{2} \int_{T_{1}}^{t_{1}}\left|p_{12}(s)\right| d s \leq m_{1} \widetilde{p}_{11}+m_{2} \widetilde{p}_{12} .
\end{array}
$$

It is clear that either $T_{2}<t_{2}$ or $T_{2}>t_{2}$ is satisfied.

Case 1: $T_{2}<t_{2}$ holds. The integration of (2.21) with $i=2$ from $T_{2}$ to $t_{2}$, on account of (1.10) and (2.25)-(2.27), implies

$$
\begin{array}{r}
M_{2}+m_{2}=-\sigma_{2} \int_{T_{2}}^{t_{2}} p_{21}(s) u_{1}\left(\tau_{21}(s)\right) d s-\sigma_{2} \int_{T_{2}}^{t_{2}} p_{22}(s) u_{2}\left(\tau_{22}(s)\right) d s \leq \\
\leq m_{1} \int_{T_{2}}^{t_{2}}\left|p_{21}(s)\right| d s+m_{2} \int_{T_{2}}^{t_{2}}\left|p_{22}(s)\right| d s \leq m_{1} \tilde{p}_{21}+m_{2} \tilde{p}_{22} .
\end{array}
$$

If $\delta_{1} m_{2} \leq \delta_{2} m_{1}$ then from $\left(2.20_{1}\right)$ and (2.29) we get

$$
M_{1}+m_{1} \leq m_{1} \tilde{p}_{11}+\frac{\delta_{2}}{\delta_{1}} m_{1} \tilde{p}_{12} \leq m_{1},
$$

which contradicts $\left(2.28_{1}\right)$. 
If $\delta_{1} m_{2}>\delta_{2} m_{1}$ then $\left(2.20_{2}\right)$ and (2.30) result in

$$
M_{2}+m_{2} \leq \frac{\delta_{1}}{\delta_{2}} m_{2} \tilde{p}_{21}+m_{2} \tilde{p}_{22} \leq m_{2},
$$

which contradicts $\left(2.28_{2}\right)$.

Case 2: $T_{2}>t_{2}$ holds. The integrations of (2.21) with $i=2$ from $a$ to $t_{2}$ and from $t_{2}$ to $T_{2}$, on account of (1.10), (2.22), and (2.25)-(2.27), yield

$$
\begin{aligned}
m_{2}=- & \sigma_{2} \int_{a}^{t_{2}} p_{21}(s) u_{1}\left(\tau_{21}(s)\right) d s-\sigma_{2} \int_{a}^{t_{2}} p_{22}(s) u_{2}\left(\tau_{22}(s)\right) d s \leq \\
& \leq m_{1} \int_{a}^{t_{2}}\left|p_{21}(s)\right| d s+m_{2} \int_{a}^{t_{2}}\left|p_{22}(s)\right| d s \leq m_{1} \tilde{p}_{21}+m_{2} \tilde{p}_{22}
\end{aligned}
$$

and

$$
\begin{aligned}
M_{2} & +m_{2}=\sigma_{2} \int_{t_{2}}^{T_{2}} p_{21}(s) u_{1}\left(\tau_{21}(s)\right) d s+\sigma_{2} \int_{t_{2}}^{T_{2}} p_{22}(s) u_{2}\left(\tau_{22}(s)\right) d s \leq \\
& \leq M_{1} \int_{t_{2}}^{T_{2}}\left|p_{21}(s)\right| d s+M_{2} \int_{t_{2}}^{T_{2}}\left|p_{22}(s)\right| d s \leq M_{1} \widetilde{p}_{21}+M_{2} \tilde{p}_{22} .
\end{aligned}
$$

If $\delta_{1} m_{2} \leq \delta_{2} m_{1}$ then from (2.201) and (2.29) we get (2.31), which contradicts $\left(2.28_{1}\right)$.

If $\delta_{1} m_{2}>\delta_{2} m_{1}$ and $\tilde{p}_{21}>0$ then $\left(2.20_{2}\right)$ and (2.32) imply

$$
m_{2}<\frac{\delta_{1}}{\delta_{2}} m_{2} \tilde{p}_{21}+m_{2} \tilde{p}_{22} \leq m_{2},
$$

which is a contradiction.

If $\delta_{1} m_{2}>\delta_{2} m_{1}$ and $\tilde{p}_{21}=0$ then $\left(2.20_{2}\right)$ and (2.33) results in

$$
M_{2}+m_{2} \leq M_{2} \tilde{p}_{22} \leq M_{2},
$$

which contradicts $\left(2.28_{2}\right)$.

The contradictions obtained prove that at least one of the functions $u_{1}$ and $u_{2}$ does not change its sign on $\left[a, \tau^{*}\right]$. We can assume without loss of generality that

$$
\sigma_{1} u_{1}(t) \geq 0 \text { for } t \in\left[a, \tau^{*}\right] .
$$

Suppose that, on the contrary, $u_{2}$ changes its sign. Then $\left(2.28_{2}\right)$ is satisfied and either $T_{2}<t_{2}$ or $T_{2}>t_{2}$ is true.

Case 1: $T_{2}<t_{2}$ holds. The integration of (2.21) with $i=2$ from $T_{2}$ to $t_{2}$, in view of (1.10), (2.202), (2.25)-(2.27), and (2.35), implies

$$
M_{2}+m_{2}=-\sigma_{2} \int_{T_{2}}^{t_{2}} p_{21}(s) u_{1}\left(\tau_{21}(s)\right) d s-\sigma_{2} \int_{T_{2}}^{t_{2}} p_{22}(s) u_{2}\left(\tau_{22}(s)\right) d s \leq
$$




$$
\leq m_{2} \int_{T_{2}}^{t_{2}}\left|p_{22}(s)\right| d s \leq m_{2}
$$

which contradicts $\left(2.28_{2}\right)$.

Case 2: $T_{2}>t_{2}$ holds. The integrations of (2.21) with $i=2$ from $t_{2}$ to $T_{2}$ and from $a$ to $t_{2}$, with respect to (1.10), (2.25)-(2.27), and (2.35), result in (2.33) and

$$
\begin{aligned}
m_{2}=-\sigma_{2} \int_{a}^{t_{2}} p_{21}(s) u_{1}\left(\tau_{21}(s)\right) d s- & \sigma_{2} \int_{a}^{t_{2}} p_{22}(s) u_{2}\left(\tau_{22}(s)\right) d s \leq \\
& \leq m_{2} \int_{a}^{t_{2}}\left|p_{22}(s)\right| d s \leq m_{2} \tilde{p}_{22} .
\end{aligned}
$$

If $\tilde{p}_{21}=0$ then from $\left(2.20_{2}\right)$ and (2.33) we get (2.34), which contradicts $\left(2.28_{2}\right)$. If $\tilde{p}_{21}>0$ then $\left(2.20_{2}\right)$ guarantees that $\tilde{p}_{22}<1$. Consequently, (2.36) implies $m_{2} \leq 0$, which contradicts $\left(2.28_{2}\right)$.

We have proved that both functions $u_{1}$ and $u_{2}$ do not change their signs on $\left[a, \tau^{*}\right]$. Let, in addition, (2.23) holds. We will show that (2.24) is satisfied. We can assume without loss of generality that $\tilde{p}_{12}>0$ and the condition (2.35) is fulfilled. Suppose that, on the contrary, (2.24) is not true. Then

$$
M_{1}>0
$$

and

$$
\sigma_{2} u_{2}(t) \leq 0 \text { for } t \in\left[a, \tau^{*}\right]
$$

Obviously, $\left(2.20_{1}\right)$ implies that

$$
\tilde{p}_{11}<1 .
$$

The integration of (2.21) with $i=1$ from $a$ to $T_{1}$, in view of (1.10), (2.22), (2.25)(2.27), and (2.38), results in

$$
\begin{aligned}
& M_{1}=\sigma_{1} \int_{a}^{T_{1}} p_{11}(s) u_{1}\left(\tau_{11}(s)\right) d s+\sigma_{1} \int_{a}^{T_{1}} p_{12}(s) u_{2}\left(\tau_{12}(s)\right) d s \leq \\
& \leq M_{1} \int_{a}^{T_{1}}\left|p_{11}(s)\right| d s \leq M_{1} \tilde{p}_{11} .
\end{aligned}
$$

Using (2.39) in the last relations, we get $M_{1} \leq 0$, which contradicts (2.37). The contradiction obtained proves that the condition (2.24) holds provided that (2.23) is satisfied.

\section{Main Results}

Recall that the numbers $\tau^{*}$ and $\tau_{i i}^{*}(i=1,2)$ are given by (1.9) and (2.1), respectively. 
Theorem 3.1. Let $\sigma_{1}, \sigma_{2} \in\{-1,1\}, i \in\{1,2\}$, and let the condition (1.10) hold. Let, moreover, there exist $\delta_{j}>0(j=1,2)$ such that

$$
\delta_{1} \int_{a}^{\tau^{*}}\left|p_{i 1}(s)\right| d s+\delta_{2} \int_{a}^{\tau^{*}}\left|p_{i 2}(s)\right| d s=\delta_{i}
$$

and

$$
\delta_{1} \int_{a}^{\tau^{*}}\left|p_{3-i 1}(s)\right| d s+\delta_{2} \int_{a}^{\tau^{*}}\left|p_{3-i 2}(s)\right| d s<\delta_{3-i} .
$$

Then the following assertions are true:

(a) If the condition (2.5) is satisfied then the operator $\ell$ given by (1.8) belongs to the set $8_{a b}^{2,\left(\sigma_{1}, \sigma_{2}\right)}(a)$.

(b) Let the condition (2.6) be satisfied. Then the operator $\ell$ given by (1.8) belongs to the set $8_{a b}^{2,\left(\sigma_{1}, \sigma_{2}\right)}(a)$ if and only if the condition (2.7) is true.

PROOF. We can assume without loss of generality that $i=1$. Let the operators $\ell$ and $\ell_{11}, \ell_{22}$ are defined by (1.8) and (2.2), respectively. We first note that (1.10) and (3.2) imply

$$
\int_{a}^{\tau_{22}^{*}} p_{22}(s) d s<1
$$

Hence, Lemma 2.2 (a) guarantees

$$
\ell_{22} \in s_{a b}(a) .
$$

Case (a). If $p_{12} \not \equiv 0$ then the assertion of the theorem follows from Proposition 3.2 in [11]. Therefore, suppose that $p_{12} \equiv 0$. Then, by virtue of (3.3) and Lemma 2.1, it is sufficient to show that $\ell_{11} \in \delta_{a b}(a)$. However, using (2.5) and Lemma 2.2 (a), we see that the inclusion $\ell_{11} \in \wp_{a b}(a)$ is true.

Case (b). According to (3.1) and (2.6), we get $p_{12} \equiv 0$. By virtue of (3.3) and Lemma 2.1, the operator $\ell$ belongs to the set $\delta_{a b}^{2,\left(\sigma_{1}, \sigma_{2}\right)}(a)$ if and only if $\ell_{11} \in$ $\delta_{a b}(a)$. However, in view of (2.6) and Lemma 2.2 (b), $\ell_{11} \in \delta_{a b}(a)$ if and only if the condition (2.7) is satisfied.

Theorem 3.2. Let $\sigma_{1}, \sigma_{2} \in\{-1,1\}$, the condition (1.10) hold, and let there exist $\delta_{j}>0(j=1,2)$ such that the relation (3.1) is satisfied for $i=1,2$. Then the following assertions are true:

(a) Let the inequality (2.3) be fulfilled and let the condition (2.5) hold for $i=$ 1,2 . Then the operator $\ell$ given by (1.8) belongs to the set $\delta_{a b}^{2,\left(\sigma_{1}, \sigma_{2}\right)}(a)$.

(b) Let the condition (2.3) hold and let there exist $i \in\{1,2\}$ such that the condition (2.6) is satisfied. Then the operator $\ell$ given by (1.8) belongs to the set $8_{a b}^{2,\left(\sigma_{1}, \sigma_{2}\right)}(a)$ if and only if the condition (2.7) holds for every $i \in\{1,2\}$ such that the condition (2.6) is true. 
(c) Let

$$
\int_{a}^{\tau^{*}} p_{12}(s) d s \int_{a}^{\tau^{*}} p_{21}(s) d s \neq 0
$$

Then the operator $\ell$ given by (1.8) belongs to the set $\delta_{a b}^{2,\left(\sigma_{1}, \sigma_{2}\right)}(a)$ if and only if there exist $i \in\{1,2\}$ such that

$$
\sum_{j=1}^{2} \int_{a}^{\tau^{*}}\left|p_{i j}(s)\right|\left(\sum_{k=1}^{2} \delta_{k} \int_{a}^{\tau_{i j}(s)}\left|p_{j k}(\xi)\right| d \xi\right) d s<\delta_{i} .
$$

PROOF. Let the operators $\ell$ and $\ell_{11}, \ell_{22}$ are defined by (1.8) and (2.2), respectively.

Case (a). According to (2.5) and Lemma 2.2 (a), we get

$$
\ell_{11} \in \mathcal{S}_{a b}(a), \quad \ell_{22} \in \wp_{a b}(a) .
$$

Hence, by virtue of (2.3) and Lemma 2.1, it is clear that $\ell \in 8_{a b}^{2,\left(\sigma_{1}, \sigma_{2}\right)}(a)$.

Case (b). It is easy to see from (1.10) and (3.1) that, for $i=1$, 2, either (2.5) or (2.6) is satisfied. Therefore, in view of (2.3), the assertion of the theorem follows immediately from Lemmas 2.1 and 2.2.

Case $(c)$. Let the operator $\ell^{*}: C\left(\left[a, \tau^{*}\right] ; \mathbb{R}^{2}\right) \rightarrow L\left(\left[a, \tau^{*}\right] ; \mathbb{R}^{2}\right)$ be defined by the formula

$$
\ell^{*}(v)(t) \stackrel{\text { def }}{=}\left(\begin{array}{l}
p_{11}(t) v_{1}\left(\tau_{11}(t)\right)+p_{12}(t) v_{2}\left(\tau_{12}(t)\right) \\
p_{21}(t) v_{1}\left(\tau_{21}(t)\right)+p_{22}(t) v_{2}\left(\tau_{22}(t)\right)
\end{array}\right)
$$

for $t \in\left[a, \tau^{*}\right]$. In the other words, $\ell^{*}$ is the restriction of $\ell$ into the space $C\left(\left[a, \tau^{*}\right] ; \mathbb{R}^{2}\right)$. Since (1.10) holds and

$$
\tau_{i k}(t) \leq \tau^{*} \quad \text { for } t \in[a, b], i, k=1,2,
$$

it is clear that $\ell \in 8_{a b}^{2,\left(\sigma_{1}, \sigma_{2}\right)}(a)$ if and only if $\ell^{*} \in 8_{a \tau^{*}}^{2,\left(\sigma_{1}, \sigma_{2}\right)}(a)$. However, according to (3.1) and Proposition 3.3 in [11], $\ell^{*} \in 8_{a \tau^{*}}^{2,\left(\sigma_{1}, \sigma_{2}\right)}(a)$ if and only if the homogeneous problem (2.21), (2.22) has only the trivial solution. Consequently, to prove the theorem it is sufficient to show that the homogeneous problem (2.21), (2.22) has only the trivial solution if and only if there exists $i \in\{1,2\}$ such that (3.5) is satisfied.

Let $u=\left(u_{1}, u_{2}\right)^{T}$ be a solution the problem (2.21), (2.22). According to (3.4) and Lemma 2.3, we can assume that

$$
\sigma_{i} u_{i}(t) \geq 0 \text { for } t \in\left[a, \tau^{*}\right], i=1,2 .
$$

Therefore, in view of (1.10) and (3.6), from (2.21) we get

$$
\sigma_{i} u_{i}(t) \leq \sigma_{i} u_{i}\left(\tau^{*}\right) \text { for } t \in\left[a, \tau^{*}\right], i=1,2 .
$$


Put

$$
\begin{aligned}
u_{i}^{*} & =\frac{\sigma_{i}}{\delta_{i}} u_{i}\left(\tau^{*}\right) \quad \text { for } i=1,2, \\
f_{i}(t) & =\sigma_{i} \sum_{k=1}^{2} \delta_{k} \int_{a}^{t}\left|p_{i k}(s)\right| d s \quad \text { for } t \in\left[a, \tau^{*}\right], i=1,2 .
\end{aligned}
$$

The integration of (2.21) from $t$ to $\tau^{*}$, on account of (1.10) and (3.7), implies

$$
\begin{aligned}
\sigma_{i} u_{i}\left(\tau^{*}\right)-\sigma_{i} u_{i}(t)= & \int_{t}^{\tau^{*}}\left|p_{i 1}(s)\right| \sigma_{1} u_{1}\left(\tau_{i 1}(s)\right) d s+\int_{t}^{\tau^{*}}\left|p_{i 2}(s)\right| \sigma_{2} u_{2}\left(\tau_{i 2}(s)\right) d s \\
& \leq \sigma_{1} u_{1}\left(\tau^{*}\right) \int_{t}^{\tau^{*}}\left|p_{i 1}(s)\right| d s+\sigma_{2} u_{2}\left(\tau^{*}\right) \int_{t}^{\tau^{*}}\left|p_{i 2}(s)\right| d s
\end{aligned}
$$

for $t \in\left[a, \tau^{*}\right], i=1,2$. Using the notation (3.8), we get

$$
\begin{aligned}
\delta_{i} u_{i}^{*}+ & \sum_{k=1}^{2} \delta_{k} u_{k}^{*} \int_{a}^{t}\left|p_{i k}(s)\right| d s \leq \\
& \leq \sigma_{i} u_{i}(t)+\sum_{k=1}^{2} \delta_{k} u_{k}^{*} \int_{a}^{\tau^{*}}\left|p_{i k}(s)\right| d s \text { for } t \in\left[a, \tau^{*}\right], i=1,2 .
\end{aligned}
$$

On the other hand, the integration of (2.21) from $a$ to $t$, in view of (1.10), (2.22), (3.7), and (3.8) yields

$$
\begin{aligned}
\sigma_{i} u_{i}(t)=\int_{a}^{t}\left|p_{i 1}(s)\right| \sigma_{1} u_{1}( & \left.\tau_{i 1}(s)\right) d s+\int_{a}^{t}\left|p_{i 2}(s)\right| \sigma_{2} u_{2}\left(\tau_{i 2}(s)\right) d s \leq \\
& \leq \delta_{1} u_{1}^{*} \int_{a}^{t}\left|p_{i 1}(s)\right| d s+\delta_{2} u_{2}^{*} \int_{a}^{t}\left|p_{i 2}(s)\right| d s
\end{aligned}
$$

for $t \in\left[a, \tau^{*}\right], i=1,2$. Now, from (3.10) and (3.11) we obtain

$$
\delta_{i} u_{i}^{*} \leq \delta_{1} u_{1}^{*} \int_{a}^{\tau^{*}}\left|p_{i 1}(s)\right| d s+\delta_{2} u_{2}^{*} \int_{a}^{\tau^{*}}\left|p_{i 2}(s)\right| d s \quad \text { for } i=1,2,
$$

whence we get

$$
u_{i}^{*}\left(\delta_{i}-\delta_{i} \int_{a}^{\tau^{*}}\left|p_{i i}(s)\right| d s\right) \leq u_{3-i}^{*} \delta_{3-i} \int_{a}^{\tau^{*}}\left|p_{i 3-i}(s)\right| d s \quad(i=1,2) .
$$

By virtue of (3.1) and (3.4), (3.12) yields $u_{i}^{*} \leq u_{3-i}^{*}$ for $i=1,2$ and thus

$$
u_{1}^{*}=u_{2}^{*}\left(:=u^{*}\right) \text {. }
$$

Now (3.10), in view of (3.1) and (3.9), yields 


$$
\begin{array}{r}
\sigma_{i} u_{i}(t) \geq u^{*} \sum_{k=1}^{2} \delta_{k} \int_{a}^{t}\left|p_{i k}(s)\right| d s+u^{*}\left(\delta_{i}-\sum_{k=1}^{2} \delta_{k} \int_{a}^{\tau^{*}}\left|p_{i k}(s)\right| d s\right) \\
=u^{*} \sigma_{i} f_{i}(t) \quad \text { for } t \in\left[a, \tau^{*}\right], i=1,2 .
\end{array}
$$

On the other hand, using (3.1), (3.9), and (3.13), we can rewrite (3.11) as

$$
\begin{aligned}
& \sigma_{i} u_{i}(t) \leq u^{*} \sum_{k=1}^{2} \delta_{k} \int_{a}^{t}\left|p_{i k}(s)\right| d s=u^{*} \sigma_{i} f_{i}(t) \\
& \qquad \text { for } t \in\left[a, \tau^{*}\right], i=1,2 .
\end{aligned}
$$

Hence, (3.14) and (3.15) result in

$$
u_{i}(t)=u^{*} f_{i}(t) \quad \text { for } t \in\left[a, \tau^{*}\right], i=1,2 .
$$

Finally, the integration of (2.21) from $a$ to $\tau^{*}$, in view of (1.10), (2.22), and (3.16), yields

$$
\begin{aligned}
\sigma_{i} u_{i}\left(\tau^{*}\right)=\int_{a}^{\tau^{*}}\left|p_{i 1}(s)\right| \sigma_{1} u_{1}\left(\tau_{i 1}(s)\right) d s & +\int_{a}^{\tau^{*}}\left|p_{i 2}(s)\right| \sigma_{2} u_{2}\left(\tau_{i 2}(s)\right) d s= \\
& =u^{*} \sum_{j=1}^{2} \int_{a}^{\tau^{*}}\left|p_{i j}(s)\right| \sigma_{j} f_{j}\left(\tau_{i j}(s)\right) d s
\end{aligned}
$$

for $t \in\left[a, \tau^{*}\right], i=1,2$, whence we get

$$
u^{*}\left[\delta_{i}-\sum_{j=1}^{2} \int_{a}^{\tau^{*}}\left|p_{i j}(s)\right|\left(\sum_{k=1}^{2} \delta_{k} \int_{a}^{\tau_{i j}(s)}\left|p_{j k}(\xi)\right| d \xi\right) d s\right]=0 \quad(i=1,2)
$$

because of the notations (3.8), (3.9), and (3.13).

We have proved that every solution $u$ of the problem (2.21), (2.22) admits the representation

$$
u(t)=u^{*} f(t) \quad \text { for } t \in\left[a, \tau^{*}\right]
$$

where $f=\left(f_{1}, f_{2}\right)^{T}$ and $u^{*}$ satisfies (3.17). Consequently, if there exists $i \in\{1,2\}$ such that (3.5) is true then the homogeneous problem (2.21), (2.22) has only the trivial solution.

It remains to show that if the condition (3.5) is not satisfied for any $i \in\{1,2\}$, i. e.,

$$
\sum_{j=1}^{2} \int_{a}^{\tau^{*}}\left|p_{i j}(s)\right| \sigma_{j} f_{j}\left(\tau_{i j}(s)\right) d s=\delta_{i} \quad \text { for } i=1,2,
$$

then the problem (2.21), (2.22) has a non-trivial solution. Indeed, (3.1) and (3.9) yield

$$
\sigma_{i} f_{i}(t) \leq \sigma_{i} f_{i}\left(\tau^{*}\right)=\delta_{i} \quad \text { for } t \in\left[a, \tau^{*}\right], i=1,2 .
$$


Therefore, using (3.1) and (3.18), it is easy to verify that

$$
\begin{gathered}
0 \leq \sum_{k=1}^{2} \int_{a}^{t}\left|p_{i k}(s)\right|\left[\delta_{k}-\sigma_{k} f_{k}\left(\tau_{i k}(s)\right)\right] d s \leq \\
\leq \sum_{k=1}^{2} \int_{a}^{\tau^{*}}\left|p_{i k}(s)\right|\left[\delta_{k}-\sigma_{k} f_{k}\left(\tau_{i k}(s)\right)\right] d s= \\
=\delta_{i}-\sum_{k=1}^{2} \int_{a}^{\tau^{*}}\left|p_{i k}(s)\right| \sigma_{k} f_{k}\left(\tau_{i k}(s)\right) d s=0
\end{gathered}
$$

for $t \in\left[a, \tau^{*}\right]$ and $i=1,2$. Hence we get

$$
f_{i}(t)=\sigma_{i} \sum_{k=1}^{2} \int_{a}^{t}\left|p_{i k}(s)\right| \sigma_{k} f_{k}\left(\tau_{i k}(s)\right) d s=\sum_{k=1}^{2} \int_{a}^{t} p_{i k}(s) f_{k}\left(\tau_{i k}(s)\right) d s
$$

for $t \in\left[a, \tau^{*}\right], i=1,2$. Consequently, $f=\left(f_{1}, f_{2}\right)^{T}$ is a non-trivial solution of problem (2.21), (2.22).

\section{REFERENCES}

[1] Dilnaya, N. AND RonTó, A.: Multistage iterations and solvability of linear Cauchy problems, Miskolc Math. Notes, 4 (2003), No. 2, 89-102.

[2] Domoshnitsky, A. AND GolTSER, YA.: One approach to study of stability of integro-differential equations, Nonlinear Anal., 47 (2001), 3885-3896.

[3] HAKL, R., KiguRAdZE, I., AND PŮŽA, B.: Upper and lower solutions of boundary value problems for functional differential equations and theorems on functional differential inequalities, Georgian Math. J., 7 (2000), No. 3, 489512.

[4] HAKL, R., Lomtatidze, A., AND PŮŽA, B.: On nonnegative solutions of first order scalar functional differential equations, Mem. Differential Equations Math. Phys., 23 (2001), 51-84.

[5] Hakl, R. And Mukhigulashvili, S.: On a boundary value problem for $n$-th order linear functional differential systems, Georgian Math. J., 12 (2005), No. 2, 229-236.

[6] Kiguradze, I. AND PŮŽA, B.: Boundary value problems for systems of linear functional differential equations, Folia Facult. Scien. Natur. Univ. Masar. Brunensis, Brno, 2003.

[7] Kiguradze, I. AND PŮŽA, B.: On boundary value problems for systems of linear functional differential equations, Czechoslovak Math. J., 47 (1997), 341373.

[8] RonTó, A.: On the initial value problem for systems of linear differential equations with argument deviations, Miskolc Math. Notes, 6 (2005), No. 1, 105-127. 
[9] Ronto, A. N.: Exact solvability conditions of the Cauchy problem for systems of linear first-order functional differential equations determined by $\left(\sigma_{1}, \sigma_{2}, \ldots, \sigma_{n} ; \tau\right)$-positive operators, Ukrain. Math. J., 55 (2003), No. 11, $1853-1884$.

[10] Schwabik, Š., TvrdÝ, M., And Vejvoda, O.: Differential and Integral Equations: Boundary Value Problems and Adjoints, Academia, Praha, 1979.

[11] ŠREMR, J.: On systems of linear functional differential inequalities, Georgian Math. J., 13 (2006), No. 3, 539-572.

\section{Author's address}

\section{Jiří Šremr:}

Mathematical Institute, ACAdemy of SCIEnCEs of the CZech Republic, ŽižKova 22,

CZ-61662 BRNo, CZECH REPUBLIC

E-mail address: sremr@ipm.cz 